\title{
Características epidemiológicas e espaciais da hanseníase no Estado do Maranhão, Brasil, 2001-2012
}

\author{
Epidemiological and spatial characteristics of the hanseniase, \\ State of Maranhão, Brazil, 2001-2012
}

\author{
Débora R M Barbosa1; Manoel G Almeida²; Ariane G dos Santos²
}

\begin{abstract}
RESUMO
Introdução: o Brasil é o segundo país em prevalência de hanseníase no mundo, fato que retifica a assertiva de se tratar de importante problema de saúde em nosso meio, cabendo políticas públicas específicas de combate à enfermidade. Objetivo: descrever e analisar espacialmente o perfil dos casos de hanseníase notificados no Estado do Maranhão. Métodos: estudo epidemiológico quantitativo de abordagem descritiva, de série histórica, através de dados do Sistema de Vigilância Epidemiológico (SVE) referentes aos casos novos de hanseníase em residentes no Maranhão, entre 2001 e 2012. A análise se deu através de estatística descritiva, tendo sido utilizados os programas Tabwin 3.2 e Microssoft Excel 2007. Resultados: dos 54.719 casos novos registrados, a maior parte era do sexo masculino (56,69\%), com idade entre 20 e 39 anos (35,17\%), pardos (52,57\%), com até 4 anos de estudo $(43,33 \%)$. A forma clínica e classe operacional mais prevalentes foram a dimorfa e multibacilar, respectivamente, esta última mais frequente no sexo masculino. A maior prevalência foi encontrada em São Luís (13,65\%) e o maior Coeficiente de Detecção foi registrado no município de Junco do Maranhão (23,27casos/10.000hab.). Conclusão: o estudo demonstrou mudanças significativas no perfil da doença no Estado no decorrer dos anos, com redução do número absoluto de casos em algumas regiões e padrões endêmicos ou hiperendêmicos em quase todo o Estado. O conhecimento das características espaciais da epidemiologia da doença possibilita a análise de suas peculiaridades e possíveis pontos de intervenção, potencializando reformas estruturantes com potencial de interferir no quadro epidemiológico da região.
\end{abstract}

Palavras-chave: Hanseníase/epidemiologia. Distribuição Espacial da População. Maranhão.

\section{Introdução}

A hanseníase é uma doença granulomatosa infectocontagiosa causada pelo bacilo Mycobacterium leprae. Esta enfermidade possui grande relevância para saúde pública em todo o mundo por sua alta incidência e prevalência em diversas regiões, tendo grande capacidade de penetração em células nervosas, onde exerce parte importante de sua imunogenicidade, acometendo principalmente pele e nervos peri-
1. Acadêmica de enfermagem do Instituto de Ensino Superior Múltiplo (IESM)

2. Acadêmico de medicina da Univeridade Federal do Piauí (UFPI)

3. Mestranda em Ciências da Saúde pela Universidade Federal do Piauí (UFPI), Professora efetiva do Instituto de Ensino Superior Múltiplo (IESM)
Endereço: rua Professor José Amável, № 770, bairro Cabral, Teresina-PI, CEP 64000.550 Aprovado para publicação em 18/03/2014 
féricos, levando a alterações da sensibilidade e deformações historicamente permeadas por estigmas e isolamento social de seus portadores. ${ }^{1}$

O Brasil ocupa o primeiro lugar no ranque de países com maior incidência e o segundo lugar na prevalência mundial de hanseníase, ficando atrás somente da Índia. ${ }^{2}$ Concentra, com efeito, $90 \%$ dos casos registrados no Continente Americano, com média de 47 mil casos novos da enfermidade a cada ano. Nota-se que nos últimos 5 anos, a maior concentração destes casos deu-se nas regiões Norte, Nordeste e Centro-Oeste do Brasil. ${ }^{3}$

A Organização Mundial de Saúde (OMS), frente ao grande número de casos no mundo, estipulou como meta aos países a redução da prevalência de hanseníase a 1 caso para cada 10.000 hab. Até o ano de 2015. Para tanto, assumiu como estratégias o diagnóstico precoce e o tratamento adequado dos casos identificados, utilizando regime de poliquimioterapia (PQT) unido a rifampicina, clofazimina e dapsona. ${ }^{4}$

Desse modo, é imprescindível o conhecimento das características epidemiológicas da hanseníase nas populações e sua distribuição espacial, de forma a possibilitar o entendimento da relação do bacilo com os indivíduos por profissionais e gestores da área da saúde e afins no sentido de ampliar investimentos de maneira estratégica e racional, tendo em vista as peculiaridades de cada região.

O estudo utiliza dados secundários, respeitando-se a confidencialidade e o anonimato dos sujeitos através de casos notificados em sistemas de informação. Os autores declaram não haver conflitos de interesse de qualquer natureza no processo de construção desta pesquisa.

Diante desse contexto, o presente trabalho tem por objetivo conhecer o perfil dos casos notificados de hanseníase ocorridos em residentes no Maranhão e sua distribuição espacial e temporal nos municípios do Estado.

\section{Material e Métodos}

\section{Caracterização da amostra}

O Maranhão é um Estado localizado no Nordeste do Brasil, entre os paralelos $1^{\circ} 01^{\prime}$ e $10^{\circ} 21^{\prime}$ sul e os meridianos $41^{\circ} 48^{\prime}$ e $48^{\circ} 50^{\prime}$ oeste; limita-se ao norte com o Oceano Atlântico e ao sul e sudoeste com o Tocantins, a oeste com o Pará e a leste com o Piauí. Possui área total de $331.937,450 \mathrm{~km}^{2}$ e uma população de 6.574.789. Esse grande Estado, com 217 municípios, tem sua população distribuída de forma he- terogênea em seu território, com densidade demográfica de $19,81 \mathrm{hab} / \mathrm{Km}^{2}{ }^{5}$

O clima do Estado varia de sub-úmido seco, com predomínio na região Sudeste, até úmido, com predomínio na região Noroeste. A grande área do Maranhão e sua localização geográfica de transição entre os climas úmido (região amazônica) e seco (região Nordeste) permitem grandes variações climáticas em seu território, bem como diferentes vegetações. Com efeito, representa área de confluência entre os biomas Cerrado, Amazônia e Caatinga, tendo formações características como: Mata de Cocais e Baixada, resultando em vários ecossistemas diferentes. ${ }^{6,7}$

\section{Tipo de estudo}

Este trabalho consiste em um estudo epidemiológico quantitativo de abordagem descritiva, retrospectivo e de série histórica, através de dados do Sistema de Vigilância Epidemiológico (SVE) referentes aos casos novos de hanseníase diagnosticados em indivíduos residentes no Maranhão entre 2001-2012.

Partiu de revisão bibliográfica ampla, de modo a possibilitar o conhecimento aprofundado da temática proposta através de trabalhos completos publicados em língua portuguesa ou espanhola na base de dados Literatura Latino-Americana e do Caribe em Ciências da Saúde (LILACS). Como critério de exclusão: optou-se por não utilizar artigos de revisão. Por critério de inclusão, optou-se por trabalhos publicados no intervalo de 1999 a 2012, artigos completos, trabalhos em língua portuguesa e espanhola.

Estudo descritivo tem por objetivo a descrição de um determinado fenômeno, população ou estabelecimento de reações entre variáveis. Tendo por principal característica a técnica de padronização de coletas de dados. ${ }^{8} \mathrm{O}$ método quantitativo, por sua vez é o procedimento sistemático para descrição e explicação de fenômenos. ${ }^{9}$ É capaz, pois, de permitir a precisão dos resultados, excluindo possíveis distorções de análise e interpretação, dando margem de segurança quanto às inferências.

\section{Variáveis de estudo}

As variáveis estudadas foram: raça, sexo, idade, escolaridade, faixa etária, Município de residência, ano do diagnóstico, tipo de entrada, tipo de saída, forma bacilífera, tipo de encerramento, número de lesões, coeficiente de detecção (CD) e mortalidade. Para a obtenção do mapa temático, os dados foram geocodificados segundo município de notificação com auxílio do programa TabWin 3.2. 


\section{Processamento e análise dos dados}

Foram calculadas as taxas de incidência por sexo e por faixa etária dividindo-se o número de casos novos de Hanseníase pela população e multiplicando-se por 10.000 , para cada ano de estudo, bem com para taxa média dos casos novos, do coeficiente de incidência e do coeficiente de mortalidade.

As tabelas foram construídas utilizando-se o programa Excel software Microsoft Office 2010. As bases populacionais por ano, sexo e faixa etária foram obtidas no SVE. As bases populacionais para os cálculos das taxas de incidência e mortalidade foram retirados de censos e projeções demográficas do Instituto Brasileiro de Geografia e Estatística (IBGE).

\section{Resultados}

No intervalo de 2001 a 2012 foram notificados no Maranhão 54.719 casos novos de hanseníase, com média de 4.559,92 casos/ano. Quanto à variável raça/ cor, foram registrados 28.457 casos $(52,57 \%)$ em in- divíduos pardos, $17,61 \%$ em brancos, $15,85 \%$ em pretos; amarelos e indígenas representaram, respectivamente, $2,01 \%$ e $0,38 \%$. Quanto à zona de residência, $37.675(68,85 \%)$ indivíduos situam-se em área urbana, $11.940(21,82 \%)$ em área rural e $381(0,70 \%)$ em ambiente periurbano, informação ignorada em $8,63 \%$ dos casos. A maior ocorrência de casos registrados foi de casos novos com $86,35 \%$. Transferência de outro país $23(0,03 \%)$, do mesmo município $(2,45 \%)$, de outros municípios $(2,70 \%)$ e de outros Estados $(2,01 \%)$. Recidiva representou, nesta série, $2,73 \%$ do total de casos notificados.

Como pode ser observado na Figura 1A, houve maior coeficiente de incidência (CI) no ano de 2005 $(\mathrm{N}=5.620 ; 10,27 \%$; $\mathrm{CI}=9,09$ casos $/ 10.000$ hab.). Por outro lado, os anos de 2011 e 2012 apresentaram queda no CI da doença, chegando a 5,25 casos por 10.000 hab. neste último ano. Houve redução de $71,43 \%$ do coeficiente de mortalidade (CM) diretamente relacionada à hanseníase, sobretudo após o ano de 2007, ano em que foi registrado seu maior valor $(\mathrm{CM}=0,19$ óbito/10.000 hab.).

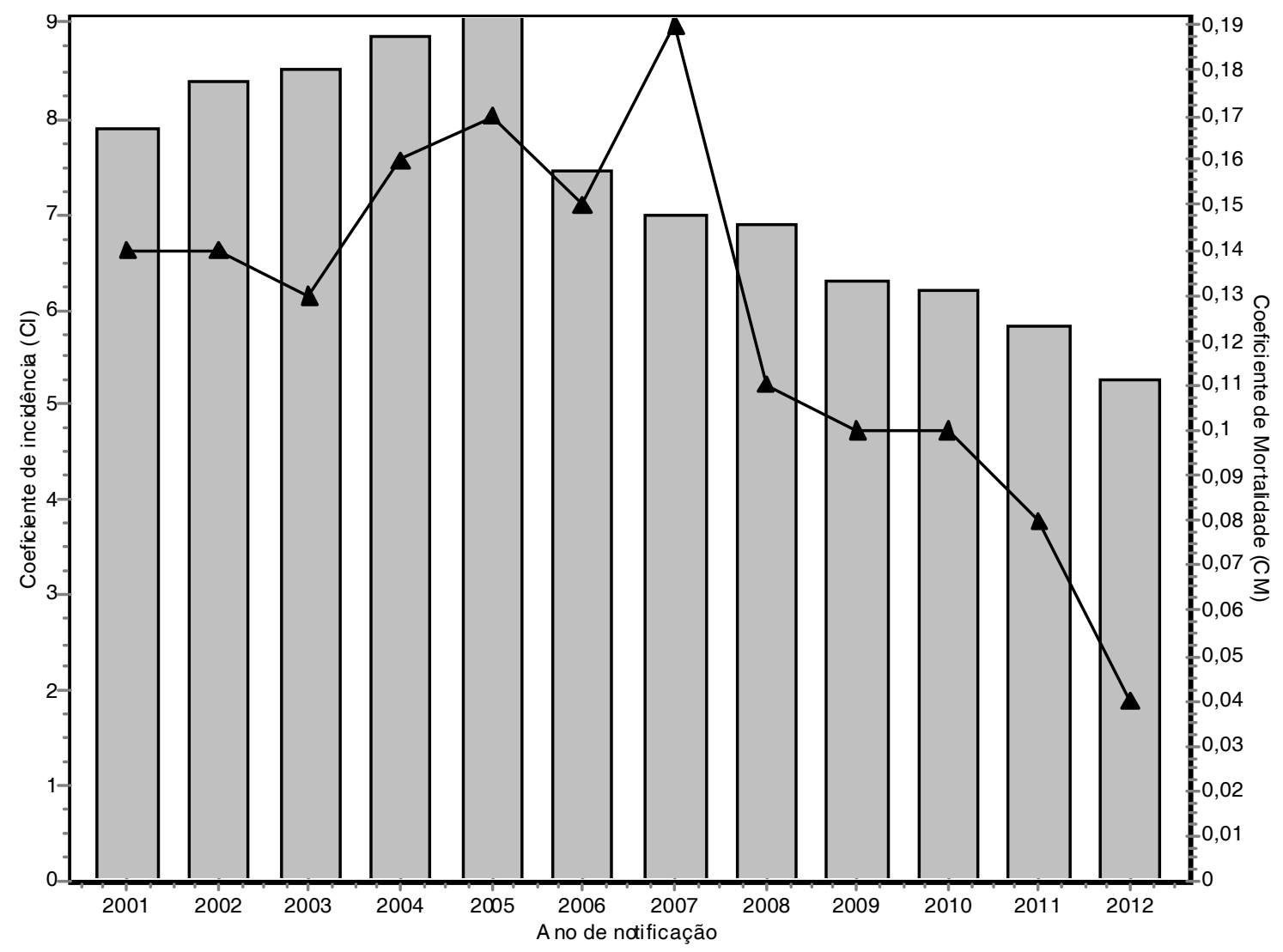

Figura 1A: Coeficientes de incidência de hanseníase e coeficiente de mortalidade de pacientes portadores de hanseníase notificada segundo ano de notificação. Maranhão, Brasil, 2001-2012. 


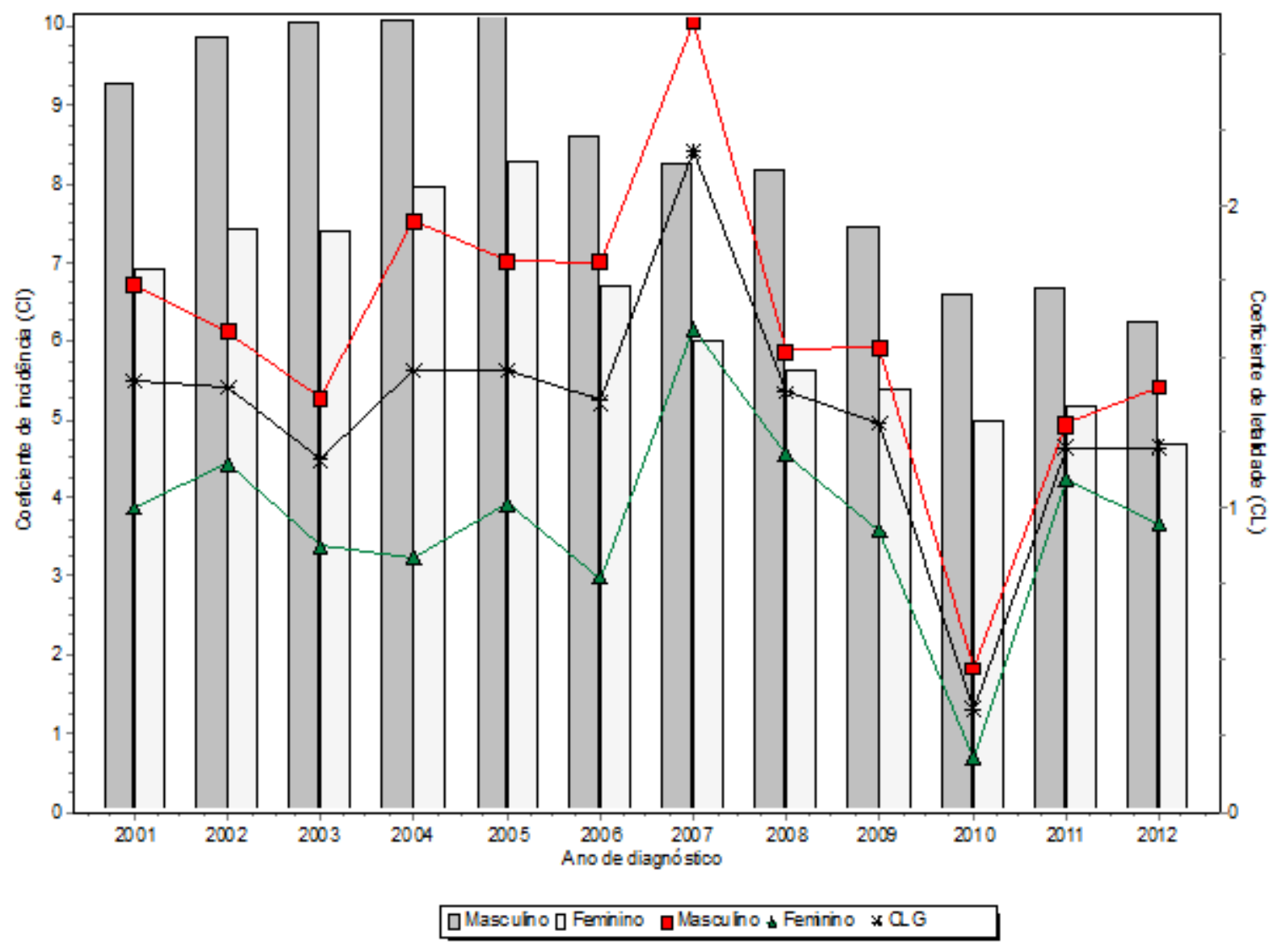

Figura 1B: Coeficientes de incidência e de letalidade geral e por sexo do paciente portador de hanseníase segundo ano de notificação. Maranhão, Brasil, 2001-2012. N: número absoluto de caso; Cl: coeficiente de incidência; CM: coeficiente de mortalidade; CL: coeficiente de letalidade; CLG: coeficiente de letalidade geral.

Foram notificados 31.021 casos de hanseníase no sexo masculino e 23.679 casos no sexo feminino $(56,69 \%$ e $43,27 \%$, respectivamente), demonstrando razão de masculinidade de 1,30:1. O sexo masculino apresentou maior CI que o sexo oposto em todos os anos deste estudo, sobretudo em indivíduos de 20 a 39 anos. Como pode ser observado na Figura 1B, os coeficientes de incidência e letalidade no sexo masculino foram maiores que no sexo oposto em todos os anos desta série e pico em 2007. O CL no sexo feminino esteve sempre inferior ao CLG e, apesar de seu crescimento de 2010 a 2011, decresceu em 2012. Por seu lado, tanto o coeficiente de incidência quanto o de mortalidade para o sexo masculino apresentaram tendência crescente nos últimos 3 anos deste estudo.

Na Figura 2 pode ser observada a distribuição de casos de hanseníase segundo faixa etária dos pacientes. Houve maior prevalência em indivíduos de
20 a $39 \operatorname{anos}(\mathrm{N}=19.039 ; 35,17 \%)$ e de 40 a $59 \operatorname{anos}$ $(\mathrm{N}=15.385 ; 28,42 \%)$, sendo escassos os casos em menores de 1 ano de idade ( $\mathrm{N}=43 ; 0,07 \%)$.

A maior ocorrência de hanseníase concentrouse em indivíduos que possuíam da $1^{\mathrm{a}}$ a $4^{\mathrm{a}}$ série incompleta do Ensino Fundamental (EF), com 12.629 casos $(23,33 \%)$, e entre a $5^{\text {a }}$ a $8^{\text {a }}$ série incompleta do EF, com 12.573 casos $(23,22 \%)$; analfabetos representaram $10.831(20,0 \%) ; 2.266(4,18 \%)$ possuíam até quatro anos de estudo (4 ${ }^{\mathrm{a}}$ série completa do EF); 1.478 casos $(2,73 \%)$ com 8 anos de escolaridade (Ensino Fundamental Completo); 8171(15,09\%) possuíram de 8 a 11 anos de escolaridade (ensino médio incompleto ou completo); 205 casos $(0,37 \%)$ possuíam educação superior incompleta e 1.320 pacientes $(2,43 \%)$ educação superior completa.

Quanto ao número de lesões, 21,63\% não apresentavam lesão ao diagnóstico e 4,96\% apresentavam 


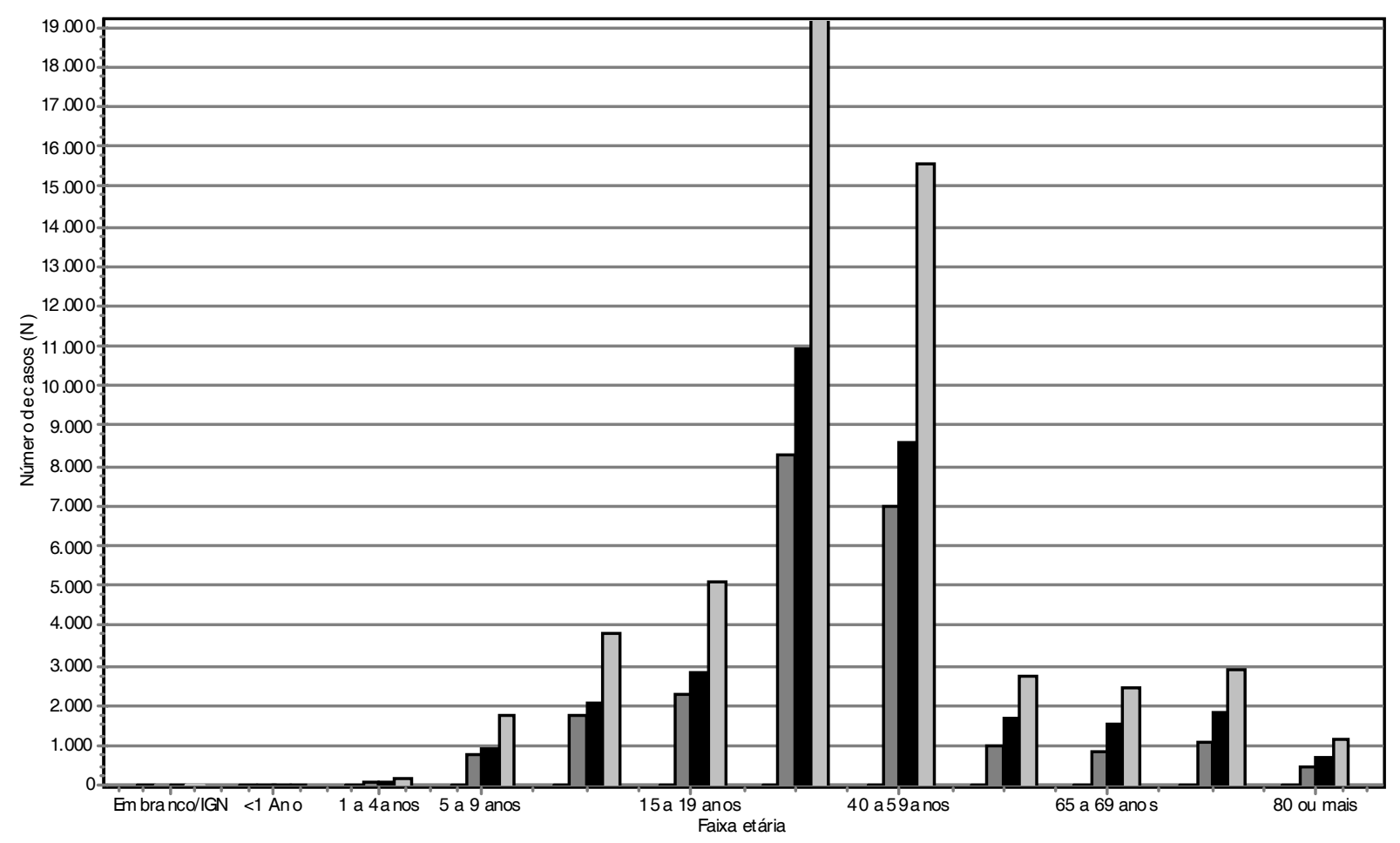

Figura 2: Distribuição de casos notificados de hanseníase segundo sexo e faixa etária dos pacientes. Maranhão, Brasil, $2001-2012$. $\mathrm{N}$ : número absoluto de casos; IGN: casos ignorados quanto à variável idade.

entre 2 e 5 lesões cutâneas. Todavia, 73,41\% dos casos notificados apresentavam, ao diagnóstico, mais de 5 lesões, sua maioria no sexo masculino $(55,37 \%)$.

A maior parte dos pacientes apresenta-se ao diagnóstico com a forma dimorfa da doença, com prevalência maior no sexo masculino de $21,79 \%$ contra $13,16 \%$ no sexo oposto. A forma virchowiana também apresentou em nossa série maior acometimento no sexo masculino $(9,89 \%)$ que no sexo feminino $(3,65 \%)$. Todas as outras formas clínicas ao diagnóstico foram mais prevalentes em mulheres que em homens, como pode ser observado na Tabela 1.

Ainda de acordo com a Tabela 1, o sexo masculino é acometido mais frequentemente com a forma multibacilar, apresentando-se com 37,90\% dos casos; em contrapartida, o sexo feminino apresentase com $20,19 \%$ do total de multibacilares e $23,02 \%$ de casos paucibacilares, valor superior ao encontrado no sexo masculino $(18,68 \%)$. Grande parte dos pacientes apresentaram, ao diagnóstico, Grau 0 de incapacidade física $(56,14 \%)$, seguido pelos que apresentaram Grau I (17,56\%) e Grau II 5,39\%).

Quanto ao tipo de saída, $71 \%$ dos pacientes evoluem para cura; $1,34 \%$ corresponderam a óbitos, $4,26 \%$ de abandono e transferidos representaram $6,89 \%$. Grande parte dos pacientes evoluiu sem incapacidade física durante o tratamento $(31,58 \%)$. No entanto, foram classificados como Graus I e II ao final do tratamento, respectivamente, $6,57 \%$ e $2,16 \%$ dos casos (Tabela 2).

Em relação aos municípios de notificação, o maior número de casos foi encontrado em São Luís $(13,65 \%)$, seguido por Imperatriz $(8,05 \%)$ e Timon $(4,67 \%)$, que delimitam área de prevalência intermediária na região central do Estado (Figura 3A).

$\mathrm{O}$ maior CD médio para esta série histórica foi encontrado na cidade de Junco do Maranhão (CD: 23,27 casos/10.000hab.), seguido de Governador Archer (CD: 20,66 casos/10.000hab.) e Itinga do Maranhão (CD: 19,25 casos/10.000hab.). Observouse concentração de municípios com maior CD médio na região central do Estado. 5,53\% dos municípios do Maranhão apresentam CD médio, $18,90 \%$ apresentam CD alto, $18,43 \%$ apresentam CD muito alto e $56,68 \%$ apresentam $\mathrm{CD}$ característico de áreas hiperendêmicas (Figura 3B). 
Tabela 1: Caracterização da hanseníase segundo sexo, forma clínica e classificação operacional grau de incapacidade física. Maranhão, Brasil, 2001-2012

\begin{tabular}{|c|c|c|c|c|c|c|c|}
\hline Sexo/forma & Ign/branco & Indeterminada & Tuberculóide & Dimorfa & Virchowiana & $\begin{array}{c}\text { Não } \\
\text { classificada }\end{array}$ & Total \\
\hline Ignorado & 7 & 6 & 3 & 0 & 3 & 0 & 19 \\
\hline Masculino & 2.788 & 4.603 & 5.060 & 11.926 & 5.413 & 1.231 & 31.021 \\
\hline Feminino & 2.051 & 5.489 & 6.104 & 7.201 & 1.995 & 839 & 23.679 \\
\hline Total & 4.846 & 10.098 & 11.167 & 19.127 & 7.411 & 2.070 & 54.719 \\
\hline \multicolumn{8}{|c|}{ Grau de incapacidade física segundo forma clínica ao diagnóstico } \\
\hline Grau/forma & Ign/branco & Indeterminada & Tuberculóide & Dimorfa & Virchowiana & $\begin{array}{c}\text { Não } \\
\text { classificada }\end{array}$ & Total \\
\hline Ignorado & 339 & 213 & 203 & 453 & 147 & 70 & 1425 \\
\hline Grau 0 & 2779 & 7463 & 7415 & 9335 & 2818 & 910 & 30720 \\
\hline Grau I & 756 & 741 & 1115 & 4521 & 2095 & 378 & 9606 \\
\hline Grau II & 258 & 103 & 242 & 1438 & 758 & 149 & 2948 \\
\hline Não avaliado & 714 & 1578 & 2192 & 3380 & 1593 & 563 & 10020 \\
\hline
\end{tabular}

Ign: casos ignorados quanto à variável analisada

Tabela 2: Caracterização da hanseníase segundo grau de incapacidade e tipo de saída. Maranhão, Brasil, 2001-2012

\begin{tabular}{|c|c|c|c|c|c|c|}
\hline \multicolumn{7}{|c|}{ Grau de incapacidade na ocasião da cura segundo tipo de saída } \\
\hline & Ign/branco & Grau 0 & Grau I & Grau II & $\begin{array}{c}\text { Não } \\
\text { avaliado }\end{array}$ & Total \\
\hline Não preenchido & 7.907 & 610 & 190 & 60 & 267 & 9.034 \\
\hline Cura & 3.877 & 16.371 & 3.298 & 1.085 & 14.220 & 38.851 \\
\hline Transferência mesmo município & 275 & 35 & 13 & 1 & 139 & 463 \\
\hline Transferência outro município & 764 & 61 & 28 & 4 & 129 & 986 \\
\hline Transferência outro Estado & 301 & 50 & 18 & 4 & 87 & 460 \\
\hline Transferência outro País & 99 & 9 & 5 & 2 & 20 & 135 \\
\hline Óbito & 558 & 42 & 21 & 9 & 102 & 732 \\
\hline Abandono & 1.856 & 100 & 21 & 16 & 340 & 2.333 \\
\hline Transferência não especificada & 1725 & 0 & 0 & 0 & 0 & 1725 \\
\hline Total & 17.362 & 17278 & 3594 & 1181 & 15304 & 54.719 \\
\hline
\end{tabular}

Ign: casos ignorados quanto à variável analisada 

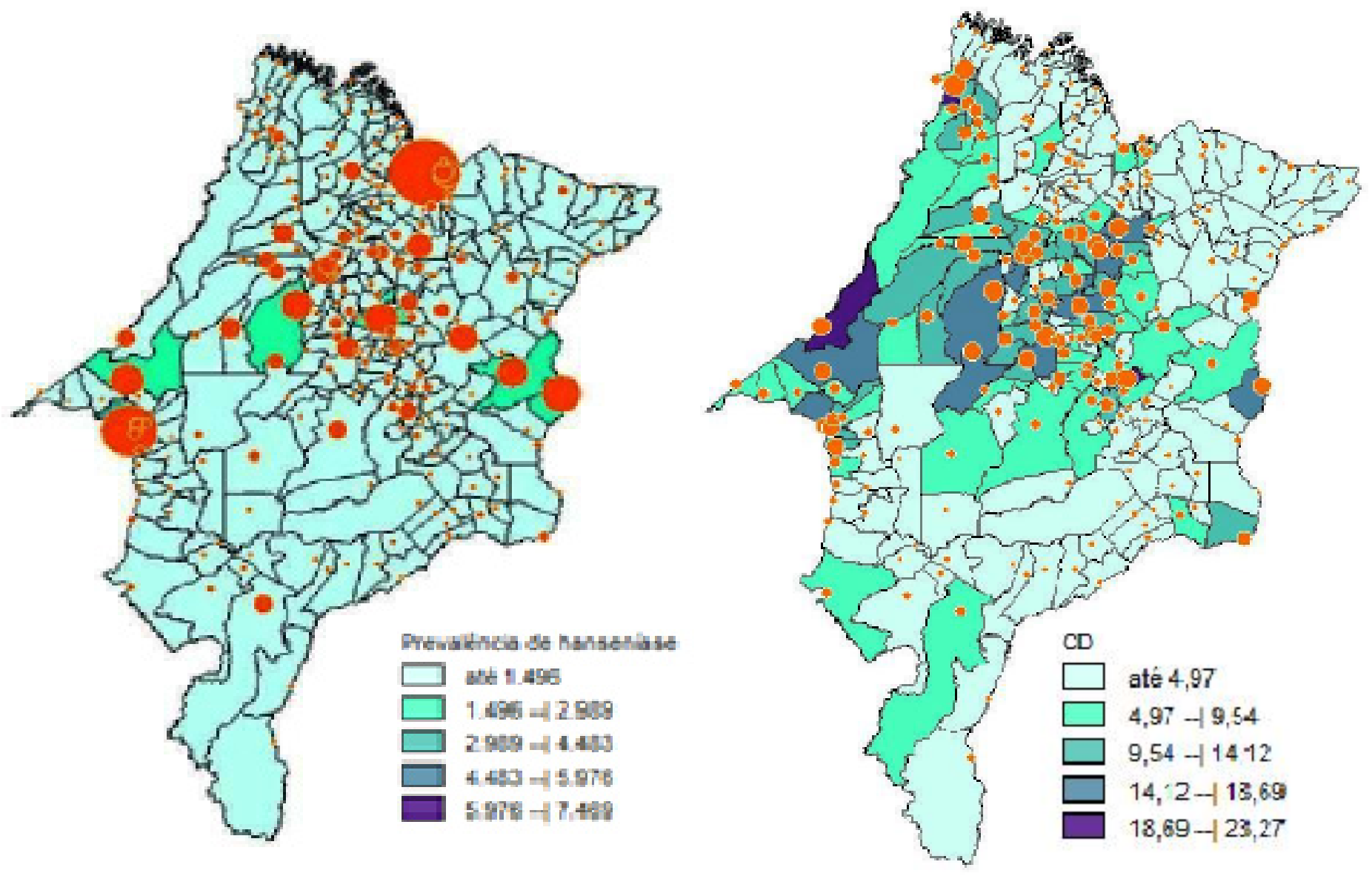

Figura 3A - Distribuição espacial de casos de hanseníase segundo município de residência do paciente. Maranhão, Brasil, $2001-2012$. Figura 3B - Distribuição espacial de casos de hanseníase segundo coeficiente de detecção (CD) por município de notificação. Maranhão, Brasil, 2001-2012.

Legenda: as áreas circulares em destaque representam a proporção relativa de casos absolutos de hanseníase por município (Fig. 3B).

\section{Discussão}

Este estudo destacou a maior prevalência de casos de hanseníase em indivíduos do sexo masculino em relação ao sexo feminino, corroborando dados nacionais e internacionais sobre o tema. ${ }^{9-10}$ Costuma-se assumir que os fatores construtores da identidade masculina condicionam aspectos importantes da epidemiologia da hanseníase e de diversas outras moléstias - infecciosas ou não - nas populações o maior cont. No que tange o gênero masculino, podese assumir como características o maior contato interhumano em locais de trabalho, menor preocupação com aspectos reacionados ao seu corpo e sua saúde, assim como a dificuldade de acesso aos serviços de públicos de saúde. Dentre outros, esses motivos levam os homens a um quadro de maior risco de adoecimento e morte. ${ }^{11}$
Quando se considera a faixa etária como variável de análise, a maior prevalência se deu entre 20 e 39 anos, intervalo que concentra a maior parte da população economicamente ativa. A hanseníase atingiu substancialmente menos crianças e adolescentes do que adultos. Esses dados estão de acordo com os encontrados na literatura nacional. ${ }^{12}$

Os dados de escolaridade encontrados neste estudo foram concordantes com diversos trabalhos similares realizados no Brasil, que relatam a baixa escolaridade como importante característica dos portadores de hanseníase. ${ }^{2,13,14}$ A baixa escolaridade da população surge em reflexo a aspectos sociais e econômicos precários, que influenciam na transmissão do agente infeccioso e no aumento da doença nas populações, haja vista as piores condições médico-sanitárias às quais estão submetidas, além de contribuir para menor adesão ao tratamento medicamentoso. 
A maior parte dos casos foi registrada em indivíduos pardos e residentes em zona urbana dos municípios. Esses valores se relacionam com a composição étnica do Estado. Segundo $\operatorname{Garcia}^{15}(2001)$, esse achado reproduz um processo histórico de colonização, mistura de raças, migração e organização espacial no espaço urbano. O processo de urbanização da hanseníase o Brasil tem se intensificado nas últimas décadas, sobretudo em reflexo a condições precárias de vida da população e ao acesso restrito aos bens e serviços coletivos, tais como escolas, serviços de educação segurança e saúde. Essas características se coadunam no espaço da cidade, onde o corpo urbano se fragiliza diante da elevada densidade populacional e vulnerabilidade socioeconômica, que determinam, em conjunto, um panorama geral de adoecimento e morte.

A maioria dos pacientes apresenta-se como casos novos. Melão cols. ${ }^{2}$, em estudo realizado em Santa Catarina (2011), Sobrinho cols. ${ }^{16}$ no Paraná (2008) e Mello cols. ${ }^{17}$ na cidade de Tubarão (2006), também em Santa Catarina, apresentaram valores semelhantes de casos novos. Esses trabalhos apresentaram porcentagens de recidiva inferiores aos apresentados neste trabalho.

As formas clínicas dimorfa e tuberculóide foram as mais frequentes no Maranhão. A prevalência de hanseníase dimórfica no Estado foi superior à encontrada por Lima cols. ${ }^{25}$ no Distrito Federal (2008) e Melão cols. ${ }^{2}$ em Tubarão (SC) dois anos antes, sendo que o segundo foi superado por valores encontrados nessas pesquisas. Dados de outros trabalhos, no entanto, apontam para a predominância de formas tardias da doença, no caso da dimorfa, corroborando com os resultados encontrados nesta série. Cabe salientar que as formas dimorfa e virchowiana foram mais prevalentes no sexo masculino. ${ }^{16,18,19}$

Quando se relaciona a classe operacional da hanseníase com o sexo dos pacientes, a maior parte dos homens apresentou a forma multibacilar e as mulheres apresentaram predominantemente a forma paucibacilar, aspectos também descritos em outros trabalhos sobre o tema. ${ }^{2,20}$

A maior parte dos pacientes não apresentou incapacidade física ao diagnóstico $(56,14 \%)$ ou a apresentou de forma moderada $(17,56 \%)$, tendo sido classificados, respectivamente, como Grau 0 e Grau I. O acometimento Grau II $(5,39 \%)$ foi inferior ao encontrado em outros estudos e à média nacional de $8,3 \%{ }^{2,9}$

As diferenças geográficas, econômicas e socioculturais no Brasil são amplas, o que se reflete na distribuição espacial da hanseníase e determina diferenças inter-regionais e interestaduais marcantes..$^{21,22}$ O coeficiente de detecção no Maranhão foi considerado alto. Contudo, a distribuição espacial dos casos de hanseníase permite a identificação de padrões geoespaciais de distribuição. No Maranhão, há maior prevalência da doença em municípios com maior número de habitantes; no entanto, há maior coeficiente de detecção em cidades menos populosas, aglutinadas no centro do triângulo imaginário composto pelas cidades com maior prevalência de hanseníase: São Luís, Imperatriz e Timon. Mais da metade $(56,68 \%)$ dos municípios do Estado são hiperendêmicos $(\mathrm{CD} \geq 4$ casos/10.000hab.) e nenhum apresentou CD baixo ( $\leq 0,2$ casos/10.000hab.).

Estudos anteriores demonstraram a importância da dinâmica espacial da epidemiologia da hanseníase, por tratar-se de doença cuja transmissão depende de contato inter-humano. ${ }^{23}$ Desse modo, áreas de alta prevalência sustentam a endemicidade de áreas circunvizinhas, o que pode ser bem observado nas Figuras 3A e 3B. Há pouca coincidência entre áreas de alta prevalência e áreas de elevado coeficiente de detecção.

O espaço geográfico, contudo, não se restringe ao relevo de determinada região, mas estende-se aos laços que os povos desenvolvem entre si e com o solo onde vivem. As desigualdades intermunicipais identificadas e as áreas de maior endemicidade devem ser entendidas de maneira integral. Estudos anteriores apontam para serem essas áreas regiões de amplas desigualdades sociais e onde historicamente já se havia sido construído espaços históricos de convivência com a hanseníase. ${ }^{21}$ Esses espaços condicionaram laços afetivos nas comunidades locais e que tanto orientam a forma de enxergar a relação saúde-doença quanto os aspectos mais objetivos da vida.

A maior parte dos pacientes evolui para cura após tratamento, vários deles com sequelas em decorrência da hanseníase. Dentre os casos notificados, 1,34\% evoluíram para óbito, valor inferior ao encontrado por Ramos cols. ${ }^{24}$ (2013) para o Estado de Mato Grosso entre os anos 2000 a 2007.

\section{Conclusão}

O presente estudo permitiu identificar o perfil da hanseníase em seus aspectos epidemiológicos no Estado do Maranhão, demonstrando mudanças significativas no perfil da doença no Estado no decorrer 
dos anos, com redução do número absoluto de casos em algumas regiões, mas manutenção do padrão hiperendêmico do quase geral na região. Esses dados apontam para a grande carga bacilífera na população e para a dificuldade que a rede de serviços de saúde, sobretudo a atenção básica, possui para diagnosticar e conduzir satisfatoriamente o indivíduo com hanseníase.

O conhecimento das características geoespaciais da doença no Maranhão permite ao gestor a ela- boração de estratégias controle da hanseníase mais específicas e racionais ao permitir o delineamento de traços gerais que determinam a maneira com que a doença recai localmente nas populações. Com efeito, este trabalho é capaz de identificar áreas críticas da endemia e abre a possibilidade de analisar o impacto de ações em saúde no Maranhão. Desse modo, criase a possibilidade de interferir com maior impacto no quadro epidemiológico da região.

\begin{abstract}
Introduction: Brazil is the second country in prevalence of leprosy in the world, a fact that rectifies the assertion it is important health problem in our country, being specific public policies to combat disease. Objective: To describe and analyze the spatial profile of leprosy cases reported in the state of Maranhão. Methods: a quantitative descriptive epidemiological study approach to time series, using data from the Epidemiological Surveillance System (EVS) for the new leprosy cases in the Maranhão residents between 2001 and 2012. They were analyzed using descriptive statistics, the Tabwin Microssoft 3.2 and Excel 2007 programs were used. Results: of the 54,719 new cases recorded, most were male $(56.69 \%)$, aged between 20 and 39 years (35.17\%), brown (52.57\%), with 4 years of study (43.33\%). The most prevalent clinical form and operating class were multibacillary borderline and, respectively, the latter more frequent in males. The highest prevalence was found in St Louis (13.65\%) and the highest detection rate was recorded in the municipality of Junco do Maranhão (23.27casos/10.000hab.). Conclusion: the study showed significant changes in the profile of the disease in the state over the years, reducing the absolute number of cases in some regions and endemic or hyperendemic standards in almost every state. The knowledge of spatial characteristics of the epidemiology of the disease enables the analysis of peculiarities and possible points of intervention, enhancing structural reforms with the potential to interfere with the epidemiological situation in the region.
\end{abstract}

Keyworks: Leprosy/epidemiology. Residence Characteristics. Maranhão.

\section{Referências}

1. Brasil. Hanseníase. In: Guia de Vigilância Epidemiológica. Brasília: MS, SVS, 2009. Disponível em: http:// portal.saude.gov.br/portal/arquivos/pdf/gve_7ed.pdf. Acessado em ago 2013.

2. Melão $S$ cols. Perfil epidemiológico da hanseníase no extremo sul de Santa Catarina. Revista da Sociedade Brasileira de Medicina Tropical 44(1):79-84, jan-fev, 2011.

3. Nunes JM cols. Hanseníase: conhecimentos e mudanças na vida das pessoas acometidas. Ciência \& Saúde Coletiva, 16(Supl. 1):1311-1318, 2011.

4. Pereira EVE cols. Perfil epidemiológico da hanseníase no município de Teresina, no período de 2001-2008. An. Bras. Dermatol. 2011;86(2):235-40.

5. IBGE. Instituto Brasileiro de Geografia e Estatística. Censo Demográfico. 2010.

6. IBAMA - Instituto do Meio Ambiente e dos Recursos Naturais Renováveis. Principais resultados quantitativos do IBAMA no Maranhão anos 2009 e 2010. São Luis: Superintendência do IBAMA no Maranhão. Mimeo. 2011.
7. Muniz FH. Efeito do manejo florestal sobre a composição florística e fitossociologia da floresta na Amazônia maranhense. In: Marlúcia Bonifácio Martins e Tadeu Gomes de Oliveira (Org.). Amazônia Maranhense: Diversidade e Conservação. Belém: MPEG, p.118-140. 2011b.

8. Gil AC. Métodos e Técnicas de pesquisa social. 5. ed. São Paulo: Atlas, 1999.

9. Who. Leprosy, global situation. Wkly Epid Rec 2008; 33:293300.

10. Veronesi R, Focaccia R. Tratado de infectologia. 2a ed. São Paulo: Atheneu; 2002.

11. Almeida MG e cols. Saúde e masculinidade: uma calamidade negligenciada. Anais do IV Congresso Internacional de Estudos sobre Diversidade Sexual e de Gênero da ABEH. 2012.

12. Gomes CCD, Pontes MAA, Gonçalves HS, Penna GO. Perfil clínico-epidemiológico dos pacientes diagnosticados. An Bras Dermatol. 2005;80(Supl 3):S283-8.

13. Martins PV, Caponi S. Hanseníase, exclusão e preconceito: histórias de vida de mulheres em Santa Catarina. Ciência \& Saúde Coletiva, 15(Supl. 1):1047-1054, 2010. 
14. Ribeiro-Junior AF, Vieira MA, Caldeira AP. Perfil epidemiológico da hanseníase em uma cidade endêmica no Norte de Minas Gerais. Rev. Bras. Clin. Med. São Paulo, 2012 julago;10(4):272-7.

15. Garcia JRL. Entre a "loucura" e a hanseníase: interfaces históricas das práticas e políticas instituídas. Hansen. Int., 26(1): 14-22, 2001.

16. Sobrinho RAS, Mathias TAF. Perspectivas de eliminação da hanseníase como problema de saúde publica no Estado do Paraná, Brasil. Cad Saúde Publica 2008; 24:303-314.

17. Mello RS, Popoaski MCP, Nunes DH. Perfil dos pacientes portadores de

Hanseníase na Região Sul do Estado de Santa Catarina no período de 01 de janeiro

de 1999 a 31 de dezembro de 2003. Arq Catarinenses Med 2006; 35:29-36.

18. Gomes CCD, Goncalves HS, Pontes MAA, Penna GO. Perfil clinico-epidemiológico

dos pacientes diagnosticados com hanseníase em um centro de referencia na Região Nordeste do Brasil. An Bras Dermatol 2005; 80 (supl 3): 283-288.

19. Amaral EP, Lana FCF. Analise espacial da Hanseníase na microrregião de Almenara, MG, Brasil. Rev Bras Enferm 2008; 61:701-707.
20. Longo JDM, Cunha RV. Perfil clínico- epidemiológico dos casos de hanseníase atendidos no hospital universitário em Campo Grande, Mato Grosso do Sul, de janeiro de 1994 a julho de 2005. Hansenol Int 2006; 31:9-14.

21. Magalhães MCC; Rojas LI. Diferenciação territorial da hanseníase no Brasil. Epidemiologia e Serviços de Saúde. Volume 16 - № 2 - abr/jun de 2007.

22. Andrade V. A Eliminação da Hanseníase no Brasil. Hansen. Int, 25(2): 177-179, 2000.

23. Duarte EC, Schneider MC, Paes-Souza R, Ramalho MW, Sardinha LV, Silva JB, Castillo-Salgado C. Epidemiologia das desigualdades em saúde no Brasil: um estudo exploratório. Brasília: OPAS; 2002.

24. Ramos ARS, Ferreira SMB, Ignott E. Óbitos por hanseníase como causa básica em residentes no Estado de Mato Grosso, Brasil, no período de 2000 a 2007. Epidemiol. Serv. Saúde, Brasília, v. 22, n. 2, June 2013.

25. Lima, MAR, Prata MO, Moreira D. Perfil da hanseníase no Distrito Federal no período de 2000 a 2005. Comun. ciênc. saúde;19(2):163-170, abr.-jun. 2008. 